\title{
Surface Crack Shape Evolution Modelling using an RMS SIF approach
}

\author{
A. Chahardehi ${ }^{1, \text { a }}$, F. P. Brennan ${ }^{1, \mathrm{~b}}$ and S. K. Han $^{2}$ \\ ${ }^{1}$ School of Engineering, Whittle Building, Cranfield University, Cranfield, Beds, MK43 0AL, UK. \\ ${ }^{2}$ Daewoo Shipbuilding and Marine Engineering Co. Ltd., Koje, Korea. \\ aa.chahardehi@cranfield.ac.uk, bf.brennan@cranfield.ac.uk.
}

\begin{abstract}
The majority of fatigue cracks in thick plate and tubular sections in structural components are two-dimensional surface cracks having significant propagation lives before becoming critical. The modelling of surface crack propagation life is important across a range of industries from power generation to offshore so that inspection, maintenance and repair strategies can be developed. Linear Elastic Fracture Mechanics based predictions are commonplace, however, unlike thin sections with associated one dimensional cracks frequently encountered in aerospace industries, crack shape or aspect ratio has a profound effect on crack front Stress Intensity Factor and any resulting Paris Law based life prediction. The two most commonly used approaches are to calculate the crack growth rate at a number of points around the crack front and to consider only surface and deepest points, calculating the relative crack growth rates. Experience using these approaches has shown that the Paris Law co-efficient as determined from plane stress specimens appears not to be applicable to crack growth in both length and depth directions. This paper examines this apparent anomaly, explaining why this discrepancy exists and suggests a practical solution using an RMS SIF approach for surface cracks that negates the need to correct the plane stress Paris law constants.
\end{abstract}

Keywords: Stress Intensity Factor; RMS SIF; Crack Shape; Crack Aspect Ratio; Paris Law; Surface Crack 


\section{Nomenclature}

$\begin{array}{ll}\text { a } & \text { surface crack depth } \\ \mathrm{C} & \text { surface crack half length } \\ \mathrm{K} & \text { Paris law coefficient } \\ \bar{K}_{x} & \text { stress intensity factor (SIF) } \\ \bar{K}_{y} & \text { averaged weight function in the ' } x \text { ' direction } \\ \mathrm{m} & \text { averaged weight function in the ' } y \text { ' direction } \\ \mathrm{N} & \text { Paris law coefficient } \\ \mathrm{RMS} & \text { number of load cycles } \\ \phi & \text { root mean square }\end{array}$




\section{Introduction}

Surface cracks account for the majority of structural fatigue failures. Cracks usually initiate from surface defects, which then develop into a part-through crack. Several observations have shown that these cracks are usually semi-elliptical in shape and that in flat specimens these cracks tend to retain a semi-elliptical shape during their growth [1,2].

For the analysis of fatigue crack growth, the Paris law [3] has proved to be a simple, accurate and robust approach where knowledge of stress intensity factor is enough to predict the growth rate of edge and through-thickness cracks. In order to analyse the growth of surface cracks under cyclic loading, it has been generally accepted to apply the Paris law for the deepest and the surface points of the crack and then assume a semielliptical shape for the crack $[2,4]$. This has been expressed mathematically in terms of the surface and the deepest points:

$$
\frac{d a}{d N}=C_{D P}\left(\Delta K_{D P}\right)^{m} \quad ; \frac{d c}{d N}=C_{S P}\left(\Delta K_{S P}\right)^{m}
$$

$C_{D P}, C_{S P}$ and $m$ are material properties and should not depend on the loading and on the geometry of the crack-component configuration.

However, as early as the 1970 s it was observed that the two material constants $C_{D P}$ and $C_{S P}$ are not equal. Corn [5] observed that small semi-circular surface cracks under either tensile or bending loads tend to retain their semi-circular shape. Yen and Pendleberry [6] suggested that assuming $C_{S P}=0.9^{m} C_{D P}$ can explain this behaviour since the SIF for the deepest point of the small semi-circular crack is about $10 \%$ lower than the SIF at the surface point.

$\mathrm{Wu}$ [7] argued that in a surface crack, if for each point on the crack front Paris law is applied as: 


$$
\frac{d a(\phi)}{d N}=C(\phi)[\Delta K(\phi)]^{m}
$$

Then by adopting Corn's observation [5], the following relation can be obtained for $C(\phi)$ :

$$
C(\phi)=\frac{C_{D P}}{\left[1+0.1 \times(1-\sin \phi)^{2}\right]^{m}}
$$

In equation $2, \phi$ denotes the parametric angle on the crack front and $C_{D P}$ corresponds to $C\left(\frac{\pi}{2}\right)$

Notwithstanding the fact that equation (2) was derived for a crack where $a=c$, i.e. a semicircular crack, Wu used this formula to predict crack shape evolution trends for different semi-elliptical surface cracks under bending and tension [7].

In order to take the above analysis one step further, the general problem of the growth of a semi-elliptical surface crack under an arbitrary loading mode can be analysed. Instead of assuming that semi-circular cracks remain semi-circular, it is assumed that semi-elliptical cracks generally tend to retain their semi-elliptical shape. The results of this general analysis confirm the fact that not only is $C(\phi)$ not constant and varies depending on $\phi$, but also that it is dependent on crack geometry and loading mode. The load-dependent nature of this coefficient makes its application questionable. Details of this analysis are given in Appendix A.

Qualitatively, the change in Paris law coefficient along the crack front can be related to change in the stress state at the crack tip, from a state of high triaxiality of stress at the deepest point, to a biaxial stress state at the surface points. Also, there is a larger plastic 
region at the crack tip on the surface of the specimen, which further proves the requirement for separate local treatment of the Paris law in the vicinity of the region.

The analysis given in Appendix A suggests that if the Paris law is applied to individual points on the crack front in surface cracks, then the coefficients are not merely material properties. It has been shown that $C(\phi)$ generally depends on the geometric parameters of the surface crack, and may also depend on loading. Therefore it has been mathematically shown that the multi-point analysis of surface cracks using Paris law is not a reliable method of fatigue life estimation. The errors caused from this approach vary depending on the particular problem and in some cases can lead to non-conservative estimates.

\section{RMS stress intensity factors}

Cruse and Besuner [8] were the first to utilise the concept of an integrated average of the stress intensity factor in what is now known as the Root Mean Square (RMS) Stress Intensity Factor (SIF). RMS SIF is defined, for the two principal growth dimensions, as:

$$
\bar{K}_{x}^{2}=\frac{1}{\Delta A_{x}} \iint_{\Delta A_{x}} K^{2}(s) d A \text { and } \bar{K}_{y}^{2}=\frac{1}{\Delta A_{y}} \iint_{\Delta A y} K^{2}(s) d A
$$

where

$$
\Delta A_{x}=\pi a_{y} \Delta a_{x} \text { and } \Delta A_{y}=\pi a_{x} \Delta a_{y}
$$

Their method involves definition of a number of characteristic dimensions (usually two) for a crack; the crack propagation being described by keeping track of these dimensions. For the crack shown in Fig. 1, these parameters are $a_{x}$ and $a_{y}$ which denote crack lengths in the two perpendicular dimensions, as shown. Cruse and Besuner [8] assumed that the coefficients of the Paris law for this type of analysis are the same as for when normal stress intensity factor values (i.e. K) are used. 

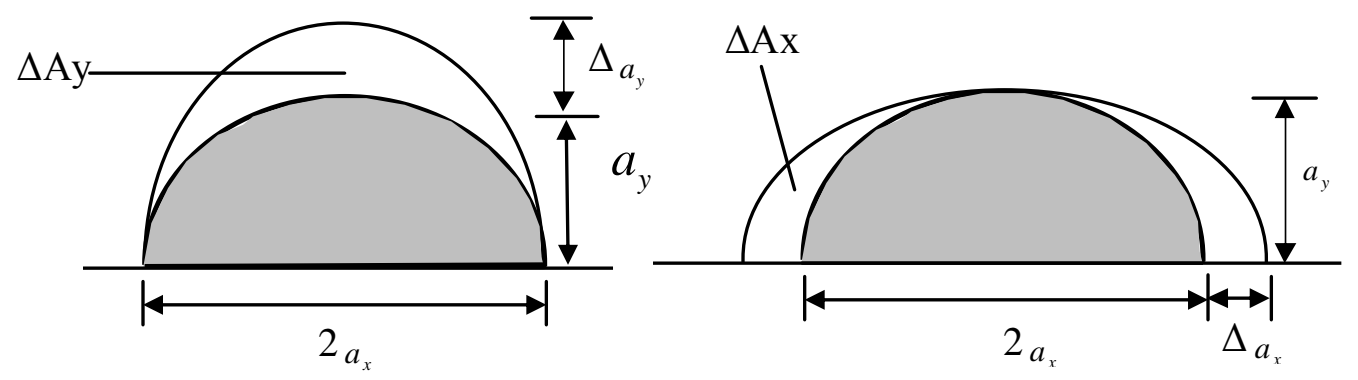

Fig. 1: Two characteristic growth dimensions

Hence the Paris law for surface crack growth can be written as:

$\frac{d a}{d N}=C_{A}\left(\Delta K_{R M S, A}\right)^{m}$ and $\frac{d c}{d N}=C_{B}\left(\Delta K_{R M S, B}\right)^{m}$

where it is postulated that $C_{A}=C_{B}$. If this relation is valid, then the problem of surface crack growth has been immensely simplified. By having two material properties ( $\mathrm{C}$ and $m$, which are independent of geometry and loading), it is only sufficient to know the average stress intensity factor values for two directions to predict the crack growth in a specific material.

Before detailing an experimental based examination of the abovementioned assumption, a few points are worth mentioning:

1) The surface point SIF value imposes a problem since at this point there is an immediate transition in the state of stress from three dimensional to biaxial. This area usually encompasses a larger plastic zone as a result of this stress state, which means that this single point does not represent the overall crack behaviour near the surface. Another 
suggested explanation is the different crack closure along the contour of the crack [9-10]. In their experiments, Kim and Song [10] observed that the crack opening ratio was about $\% 10$ greater at the deepest point than at the surface.

2) It was shown that $C(\phi)$ varies and is a function of crack geometry and loading. An averaged stress intensity factor, when used with the newly proposed form of the Paris law, would essentially eliminate these variations.

3) This new method intrinsically ensures that cracks retain their semi-elliptical shape, and no extra geometrical considerations are required.

4) Assuming a two-point approach to the crack growth problem effectively results in ignoring the value of stress intensity factor at other points along the crack front. Different loadings lead to different stress intensity factor variations, and two different loadings may result in the same SIF values at the deepest and surface points for the two specimens respectively. This, however, does not necessarily mean that the two cracks should evolve identically.

Mahmoud [11,12] carried out extensive analyses of various surface crack growth data using different techniques. The experiments' data consisted of 58 cracks from 20 tensile [11] and 50 cracks from 15 references in bending plates [12], in order to identify the most accurate method. He concluded that whereas using the average value of SIF leads to more accurate predictions in tension, for specimens under bending it is the local SIF approach that yields more accurate results.

The following section details experiments carried out to examine whether the Paris law coefficient in the two directions are identical. These experiments also show that contrary to Mahmoud's conclusion, using the averaged SIF approach leads to better predictions for specimens under cyclic bending. 


\section{Experimental study of surface crack growth under cyclic uniform loading}

\subsection{Test procedure}

For the study of the behaviour of surface cracks under fatigue conditions and to investigate the applicability of the RMS SIF concept in modelling crack growth, two tests were carried out in which surface cracks were grown into plate steel specimens from a starter surface notch. Both specimens were tested under uniform pure bending cyclic load. The only difference between the two tests was the initial aspect ratio of the starter crack. In addition, two Compact Tension (CT) tests were carried out to obtain the Paris law coefficients of the material. The specimen material in all cases was offshore steel BS7191 355D. Fig. 2 shows the data obtained from the CT tests.

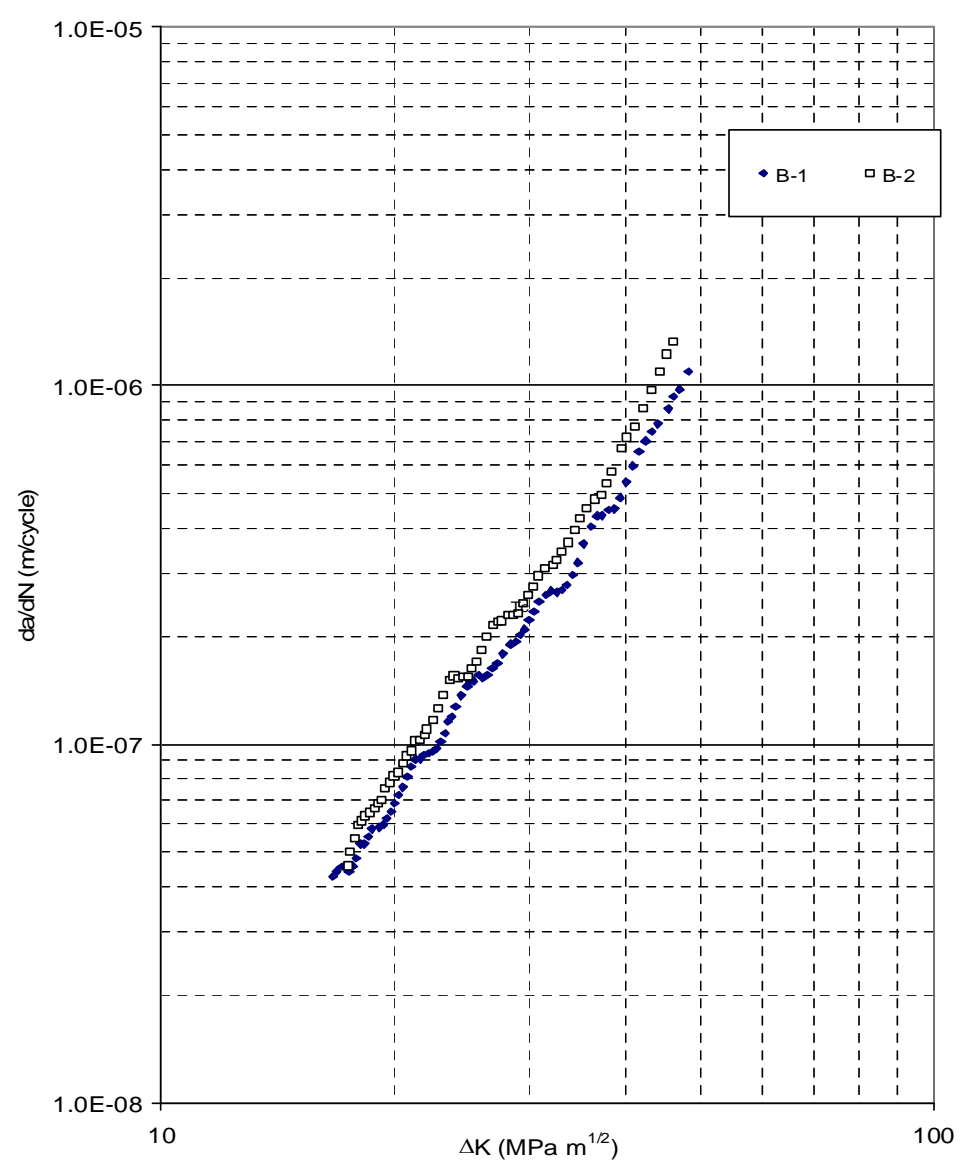

Fig. 2: CT Test Results 


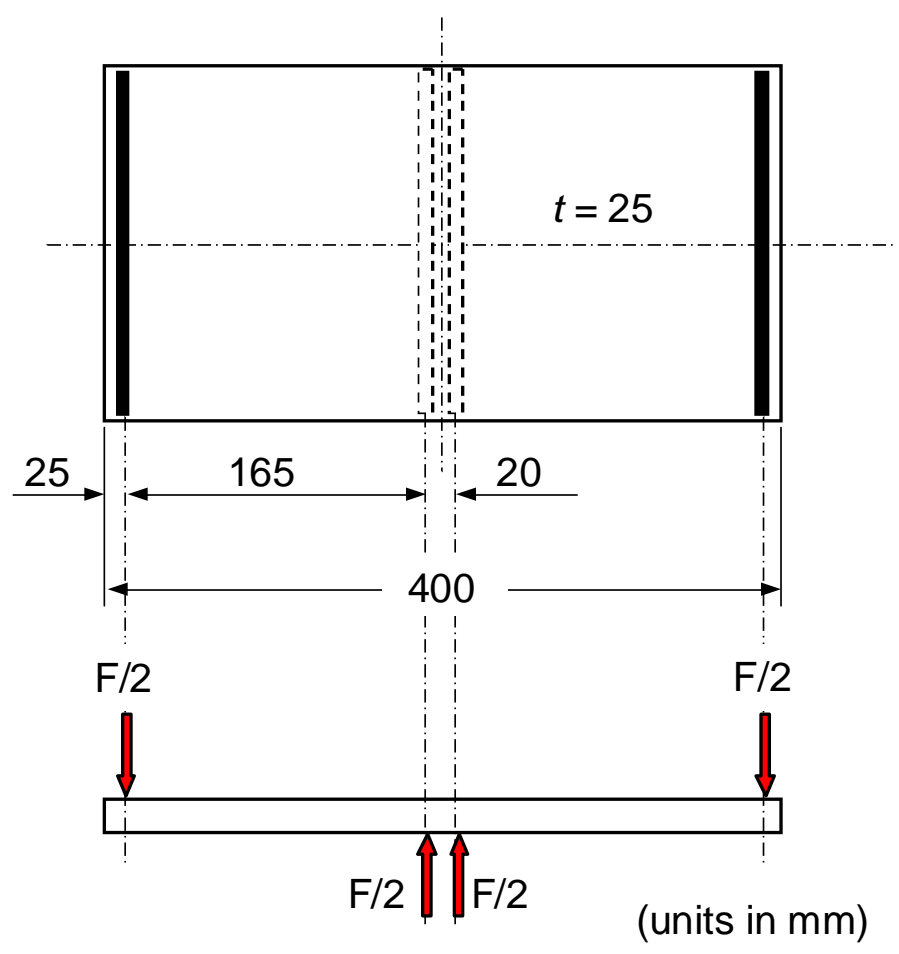

Fig. 3: Configuration of the Surface Crack Bending Tests

Fig. 3 shows the loading setup of the tests for growing surface cracks. The specimens were strain gauged to verify the pure bending stress distribution. The stress intensity factors were determined from Newman and Raju's formula [13], as a function of the crack geometry:

$K_{I}=H S_{b} \sqrt{\pi \frac{a}{Q}} F\left(\frac{a}{t}, \frac{a}{c}, \frac{c}{b}, \phi\right)$

Here $F$ is a given function of the crack/specimen geometry $(a, c, t, b)$ for tensile and bending loading modes.

From equation (3), after geometrical considerations, the RMS stress intensity factors can be evaluated as: 
$K_{R M S_{A}}{ }^{2}=\frac{2}{\pi} \int_{0}^{\pi} K^{2}(\phi) \sin ^{2}(\phi) d \phi \quad$ and $\quad K_{R M S_{B}}{ }^{2}=\frac{2}{\pi} \int_{0}^{\pi} K^{2}(\phi) \cos ^{2}(\phi) d \phi$

which is now in a form appropriate for substitution from equation (5).

Beach marks were generated to record the crack shape evolution during each test. This was achieved by increasing the stress ratio $R$ from 0.1 during primary crack growth to 0.6 for beach mark generation. These beach marks are shown in Fig. 4.

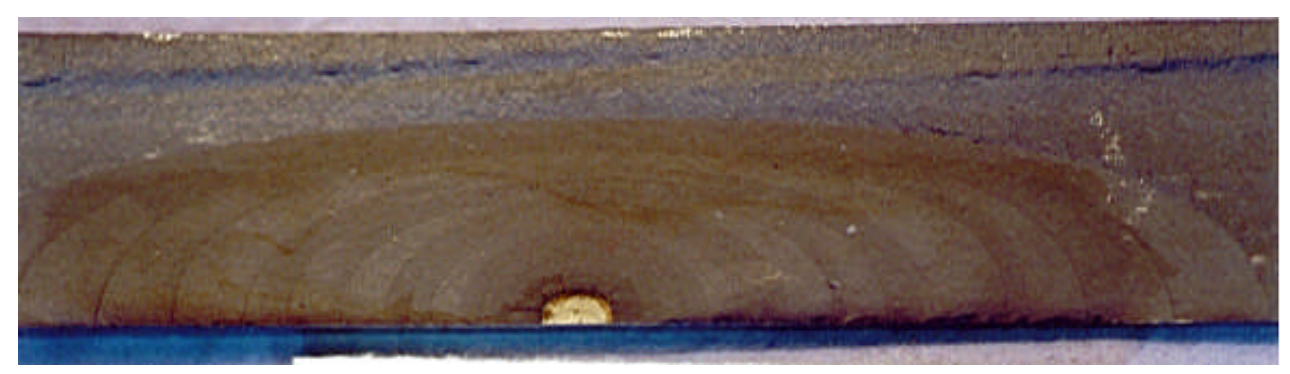

Fig 4: Beach markings on the specimens

The specimens were prepared carefully taking account of the rolling direction before introduction of the starter notches. Sinusoidal cyclic loading was applied by a servohydraulic fatigue test machine at a frequency of $2.0 \mathrm{~Hz}$. The test setup is shown in Fig 5. 


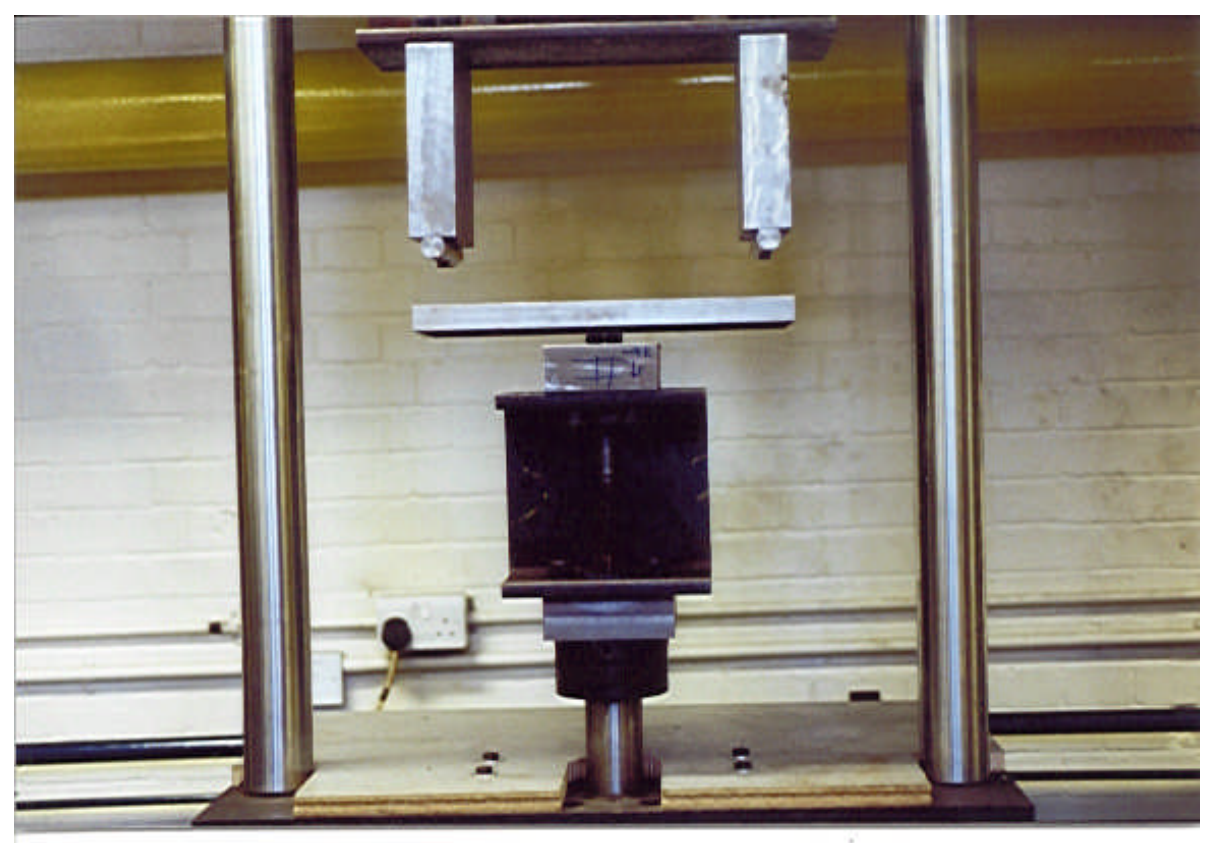

Fig 5: The test setup

\subsection{Results and analysis}

Crack growth curves in the form of crack depth $(a)$ and crack half length $(c)$ versus the number of cycles $(N)$ were obtained as a basic output from the tests. A travelling microscope with an accuracy of $\pm 0.001 \mathrm{~mm}$ was used for the measurement of the depth and length of the crack from the beach marks. The results of these analyses are shown in Fig. 6 which shows the crack growth rates as a function of stress intensity values for the deepest and surface points. $K$ values were calculated from the Newman and Raju [13] formula (equation (5)). It is clearly evident from the results of the two tests that the Paris law coefficients are different for the surface and the deepest points; the surface point is shown to have a higher resistance to fatigue crack growth than the deepest point. This set of tests clearly illustrates and confirms the argument set out in the previous section that the Paris law coefficients are not constant as long as the point SIF values are used for crack growth modelling. 


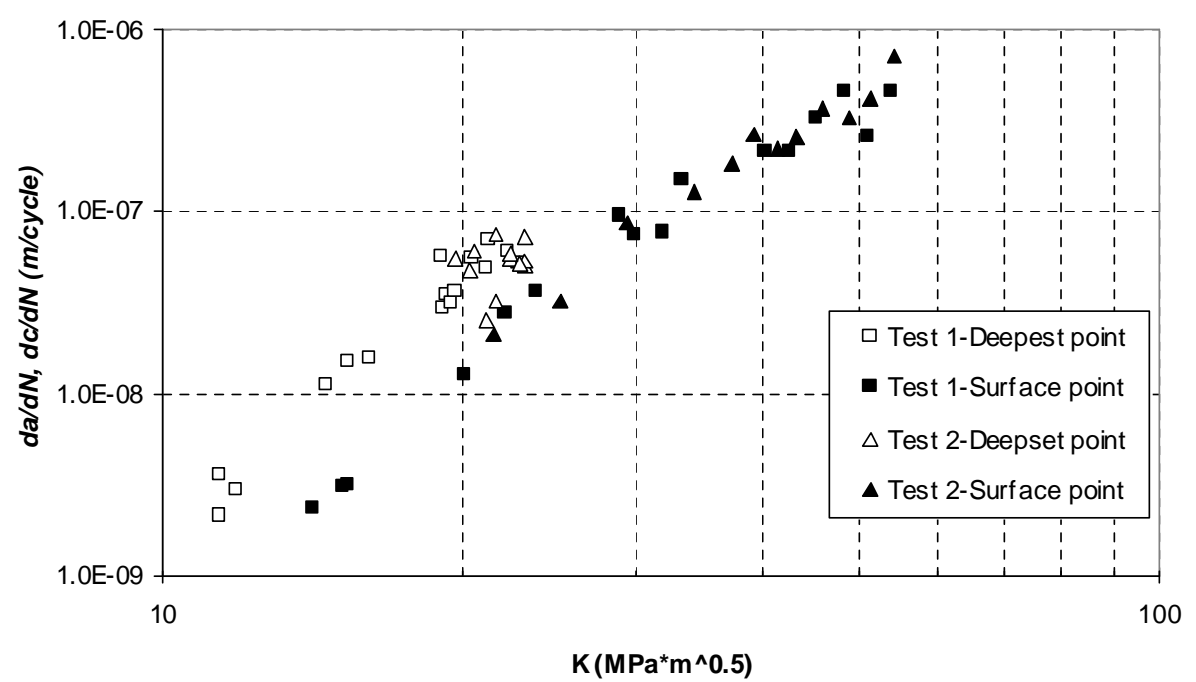

Fig 6: SIF versus crack growth rate for the dee pest and surface points

The same data obtained from the tests can be analysed differently. Fig. 7 shows the crack growth rates versus RMS values of SIF in the two different directions (depth and length).

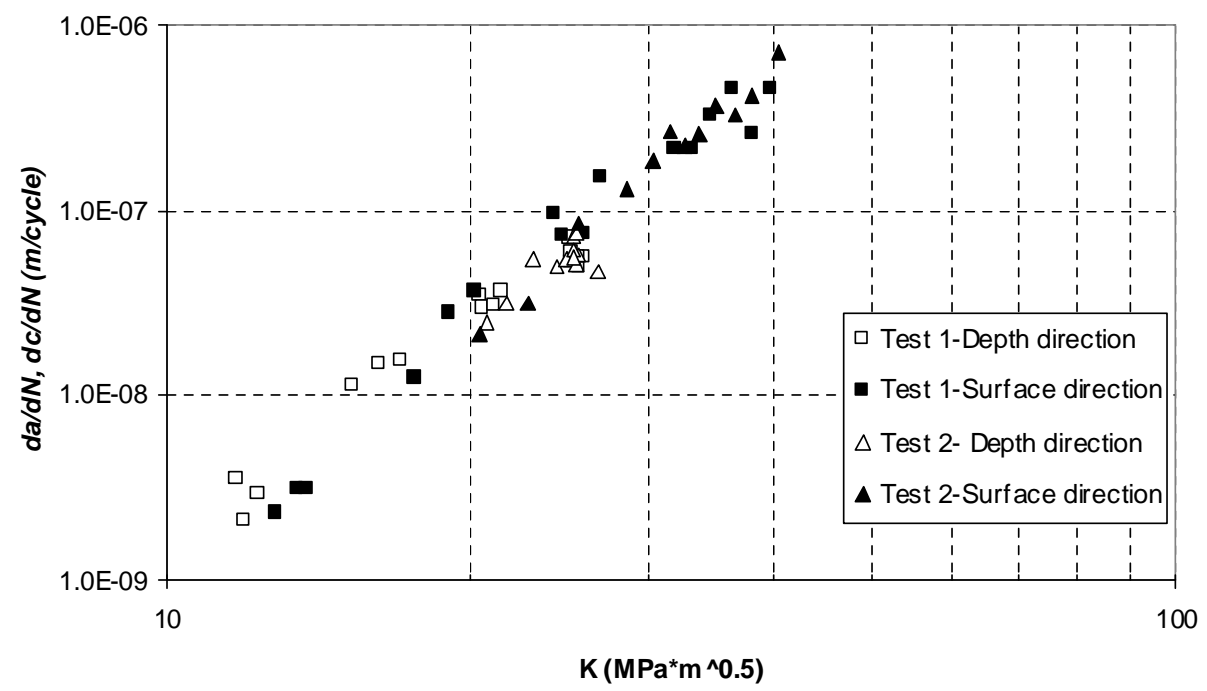

Fig 7: RMS SIF versus crack growth rates for the depth and surface directions 
Fig. 7 shows that both sets of data converge to a single line when plotted as RMS SIF compared to the quite distinct difference between the surface and deepest points in Fig. 6 . The main effect of the RMS SIF is to reduce the influence of the surface point by averaging the SIF over the crack front in the length direction. This reduces the anomaly created by the biaxial stress and relatively large plastic zone in surface and near surface points. It is suggested that this RMS SIF is more representative of the bulk crack behaviour, as growth behaviour at discrete points along a crack front are dependent on the behaviour of the crack as a whole rather than acting in isolation. The RMS SIF approach appears to allow the accurate modelling of a surface crack using the modified Paris law. Contrary to the conventional Paris law, in this method the coefficients for the surface and depth directions are the same. This observation should be further validated by additional testing; however the limited tests conducted here appear to support the theoretical explanation of the anomalous Paris law behaviour within two-dimensional surface cracks.

\section{Summary and Conclusions}

This paper examined the theoretical basis for applying the Paris law to two-dimensional surface cracks. It was shown that the Paris law coefficient could not be constant in the same material if both the deepest and surface crack points are considered. An RMS SIF approach was considered for surface cracks focusing in particular on its use with the Paris law. The RMS SIF is a relatively simple but powerful method of representing two-

dimensional crack growth in complex geometries and stress systems and promises to simplify difficult situations such as crack growth through residual stress fields [14].

The theoretical basis that the same Paris law coefficient could be used for both length and depth directions with RMS SIFs appears to be supported by the limited tests reported here. It is recommended that further testing is carried out to verify this characteristic of the RMS SIF. 


\section{Appendix A}

\section{A study of the growth of semi-elliptical cracks}

Assumptions:

1) It is assumed that semi-elliptical cracks, under the action of cyclic tensile loading, generally remain semi-elliptical.

2) It is assumed that incremental growth of the crack at points on the crack front is perpendicular to the crack front.

Fig A.1 shows a semi-elliptical crack $C_{1}$ which, under the action of cyclic loading, has grown into $C_{2} . \Delta a$ and $\Delta c$ denote the growth increments respectively in the depth and half-width of the crack. Point $P$ is an arbitrary point on $C_{1}$ with a parametric angle of $\phi$, corresponding to point $P^{\prime}$ on $C_{2}$.

Starting from the general equation of an ellipse,

$$
\left(\frac{x_{p}}{a}\right)^{2}+\left(\frac{y_{p}}{c}\right)^{2}=1 \text { and }\left(\frac{x_{p^{\prime}}}{a+\Delta a}\right)^{2}+\left(\frac{y_{p^{\prime}}}{c+\Delta c}\right)^{2}=1
$$

$\lambda$ is the angle between the $x$-axis and $\overline{P P^{\prime}}$ (the normal to the ellipse at $P$ ), and can be evaluated as a function of $\phi$ as

$$
\lambda=\tan ^{-1}\left(\frac{c}{a} \cot \phi\right)
$$

The Paris law can be written as follows for point $P$ : 
$\frac{d a(\phi)}{d N}=C(\phi)[K(\phi)]^{m}$

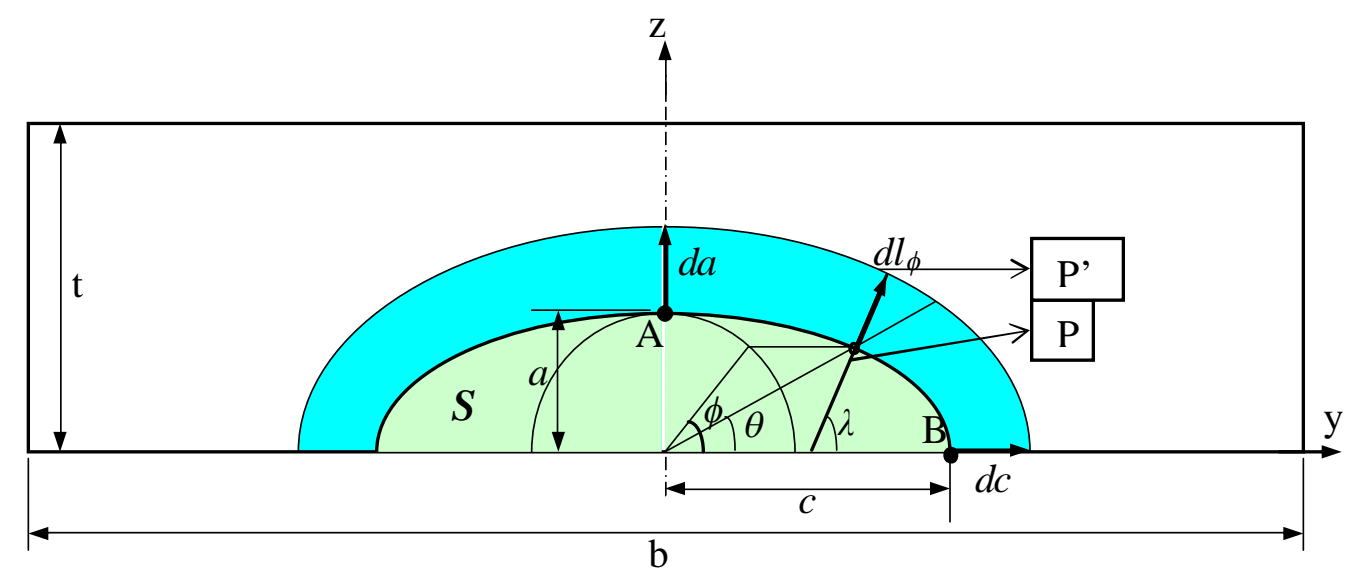

Fig. A.1. Geometrical parameters of the crack

And for the surface point (point B) as:

$\frac{d a}{d N}=C(0)[K(0)]^{m}$

Dividing equation (A-3) by equation (A-4) eliminates $d N$ as:

$\frac{C(\phi)}{C(0)}=\frac{d a(\phi)}{d a} \times\left[\frac{K(0)}{K(\phi)}\right]^{m}$

And for the deepest point:

$\frac{C(\phi)}{C(\pi / 2)}=\frac{d a(\phi)}{d c} \times\left[\frac{K(\pi / 2)}{K(\phi)}\right]^{m}$ 
If the right hand side expression of equation (A-5) and (A-6) is proven not be constant, then it is evident that $C(\phi)$ is not constant.

In order to evaluate the first ratio on the right hand side of equation (A-5), it is first noted that for small increments of crack growth $d a(\phi) \approx \Delta a(\phi)=\overline{P P^{\prime}}, d a \approx \Delta a$ and $d c \approx \Delta c$. To express $\overline{P P^{\prime}}$ as a function of $\Delta a$ and $\Delta c$, equation (A-1) can be used:

$\left(\frac{x_{p^{\prime}}}{a+\Delta a}\right)^{2}+\left(\frac{y_{p^{\prime}}}{c+\Delta c}\right)^{2}=1$

From Fig. A.1 it can be observed that

$x_{P^{\prime}}=x_{P}+\overline{P P^{\prime}} \cos \lambda=a \cos \phi+\overline{P P^{\prime}} \cos \lambda$

and similarly

$y_{P^{\prime}}=y_{P}+\overline{P P^{\prime}} \sin \lambda=c \sin \phi+\overline{P P^{\prime}} \sin \lambda$

Substituting these values in equation (A-1), and rearranging, gives:

$A \times{\overline{P P^{\prime}}}^{2}+B \times \overline{P P^{\prime}}+C=0$

where

$$
\begin{aligned}
& A=\left(\frac{1}{a+\Delta a}\right)^{2} \times \cos ^{2} \lambda+\left(\frac{1}{c+\Delta c}\right)^{2} \times \sin ^{2} \lambda \\
& B=2 a \cos \phi \cos \lambda \times\left(\frac{1}{a+\Delta a}\right)^{2}+2 c \sin \phi \sin \lambda \times\left(\frac{1}{c+\Delta c}\right)^{2}
\end{aligned}
$$


$C=a^{2} \cos ^{2} \phi \times\left(\frac{1}{a+\Delta a}\right)^{2}+c^{2} \sin ^{2} \phi \times\left(\frac{1}{c+\Delta c}\right)^{2}-1$

Equation (A-7) gives $\overline{P P^{\prime}}$ as a function of

$\overline{P P^{\prime}}=f(a, c, \Delta a, \Delta c, \phi, \lambda)$

Bearing in mind that $\lambda$ itself is a function of $a, c$, and $\phi$ (A-2), this can be simplified as:

$\overline{P P^{\prime}}=f(a, c, \Delta a, \Delta c, \phi)$

Now from equations (A-5), (A-6) and (A-8) one can get

$$
\frac{C(\phi)}{C(0)}=\frac{f(a, c, \Delta a, \Delta c, \phi)}{\Delta a} \times\left[\frac{K(0)}{K(\phi)}\right]^{m}
$$

and

$$
\frac{C(\phi)}{C(\pi / 2)}=\frac{f(a, c, \Delta a, \Delta c, \phi)}{\Delta c} \times\left[\frac{K(\pi / 2)}{K(\phi)}\right]^{m}
$$

Both these equations show that, without a need to solve equation (A-7), in a general case $C(\phi)$ is not constant and can be written as:

$C(P)=C(a, c, \phi, K(\phi))$

$K=K(\phi$, loading $)$

$C(P)=C(a, c, \phi, K(\phi$, loading $))$ 
$C(P)=C(a, c, \phi$, loading $)$

It should be noted that, from this analysis it is deduced that writing $C$ in the form of $C(\phi)$ is not accurate and could be misleading.

The significance of equation (A-12) is that it introduces a novel concept, namely that the Paris law coefficient $C$ at any point, not only depends on the position of the point ( $C$ is a function of $\phi$ which signifies spatial variability), and on the crack shape ( $a$ and $c$ ), but also on the loading $(K(\phi))$. Previous authors $[5,13,7]$ have emphasised the dependence on geometry but not the load-dependant nature of $C$. Whereas the dependence of $C$ on $\phi, a$ and $c$ could be established with relative ease (which may imply the theoretical possibility of a modified Paris law which takes these variations into account), the load dependence nature of this parameter suggests it is not possible to use the Paris law for the deepest and surface (or indeed any two) points along the crack face, since the errors resulting from this source can not be usually (and certainly not easily) evaluated. 


\section{References}

[1] Cruse T A, Meyers G J, Wilson R B, Fatigue growth of surface cracks, Flaw Growth and Fracture, ASTM STP 631 (January 1977).

[2] Muller H M, Muller S, Munz D and Neumann J, Extension of surface cracks during cyclic loading, Fracture Mechanics: Seventeenth Volume; ASTM STP 905, pp. 625643 (1986).

[3] Paris P C and Erdogan F, A critical analysis of crack propagation laws, Journal of Basic Engineering, Vol. 85, pp. 528-534 (December 1963).

[4] Mattheck C, Morawietz P and Munz D, Stress intensity factor at the surface and at the deepest point of a semi-elliptical surface crack in plates under stress gradients, International Journal of Fracture, Vol.23, No. 3, pp. 201-212 (November 1983).

[5] Corn D L, A study of cracking techniques for obtaining partial thickness cracks of pre-selected depths and shapes, Engineering Fracture Mechanics, Vol. 3, pp. 45-52 (1971).

[6] Yen C S and Pendleberry S L, Techniques for making shallow cracks in sheet metals, Materials Research Standard, November 913 (1962).

[7] Wu Shang-Xian, Shape change of surface crack during fatigue growth, Engineering Fracture Mechanics, Vol. 22, No. 5, pp. $897-913$ (1985).

[8] Cruse T A and Besuner P M, Residual life prediction for surface cracks in complex structural details, Journal of Aircraft, Vol. 12, No. 4 (April 1975).

[9] Putra I S and Schijve J, Crack opening stress measurements of surface cracks in 7075-T6 aluminium alloy plate specimen thorugh electron fractography, Fatigue and Fracture of Engineering Materials and Structures, Vol. 15, pp. 323-338 (1992).

[10] Kim J H and Song J H, Crack growth and closure behaviour of surface cracks under axial loading, Fatigue and Fracture of Engineering Materials and Structures, Vol. 15, pp. 477-489.

[11] Mahmoud M A, Quantitative prediction of growth patterns of surface fatigue cracks in tension plates, Engineering Fracture Mechanics, Vol. 31, No. 6, pp. 735-746 (1986). 
[12] Mahmoud M A, Growth patterns of surface fatigue cracks under cyclic bending- a quantitative analysis, Engineering Fracture Mechanics, Vol. 31, No. 2, pp. 357-369 (1986).

[13] Newman J C and Raju I S, An empirical stress-intensity factor equation for the surface crack, Engineering Fracture Mechanics, Vol. 15, No. 1-2, pp. 185-192 (1981).

[14] Brennan F P, Ngiam S S and Lee C W, Fatigue Crack Shape Control under Bending by Cold Working, Engineering Fracture Mechanics, Vol. 75, No. 3-4, pp 355-363 (2007). 